\title{
Endothelial Plaques as Sign of Hyphae Infiltration of Descemet's Membrane in Fungal Keratitis
}

\author{
Xiaolin Qi, ${ }^{1}$ Ting Liu, ${ }^{2}$ Man Du, ${ }^{1}$ and Hua Gao $\mathbb{D}^{1,2}$ \\ ${ }^{1}$ Eye Hospital of Shandong First Medical University, State Key Laboratory Cultivation Base, \\ Shandong Provincial Key Laboratory of Ophthalmology, Shandong Eye Institute, \\ Shandong First Medical University and Shandong Academy of Medical Sciences, Jinan, China \\ ${ }^{2}$ State Key Laboratory Cultivation Base, Shandong Provincial Key Laboratory of Ophthalmology, Shandong Eye Institute, \\ Shandong First Medical University and Shandong Academy of Medical Sciences, Qingdao, China \\ Correspondence should be addressed to Hua Gao; gaohua100@126.com
}

Received 14 February 2020; Revised 3 April 2020; Accepted 12 May 2020; Published 26 May 2020

Guest Editor: Karim Mohamed-Noriega

Copyright (c) 2020 Xiaolin Qi et al. This is an open access article distributed under the Creative Commons Attribution License, which permits unrestricted use, distribution, and reproduction in any medium, provided the original work is properly cited.

Background. To evaluate the relationship between corneal endothelial plaques and fungal hyphae infiltration in fungal keratitis. Methods. Retrospective cross-sectional study of 60 fungal keratitis patients who underwent keratoplasty between January 2013 and March 2017. The endothelial plaques were graded as follows: grade 1, 1-3 endothelial plaques; grade 2, 4-8 endothelial plaques; and grade 3, more than 8 endothelial plaques or dense, merging endothelial plaques. The fungal pathogen culture and histopathology of diseased Descemet's membrane were evaluated. Results. According to endothelial plaque grading, 3 patients were grade 1, 29 patients were grade 2, and 28 patients were grade 3 . The PK surgery was performed in 57 patients with endothelial plaques of grade 2 and grade 3 and DALK surgery in 3 patients of grade 1. The predominating fungal pathogens were Aspergillus species (63.2\%). All 57 patients with grade 2 and grade 3 had fungal hyphae in Descemet's membrane based on calcofluor white staining or PAS staining. In patients with grade 3, more hyphae and inflammatory cells were found in Descemet's membrane. The immunohistochemical staining of endothelial plaques revealed that CD15 and CD68 were positive in most cells. During the follow-up, 2 out of 3 patients who underwent DALK had recurrent fungal keratitis. Conclusions. Endothelial plaques are considered as a sign of hyphae infiltrating Descemet's membrane. PK should be performed once plaques are detected in endothelium during the surgery.

\section{Introduction}

Fungal keratitis (FK) is a severe infectious corneal disease in developing countries [1-3]. In China, more than $50 \%$ of infectious keratitis cases are the result of a fungal infection [4]. Clinical manifestations of fungal keratitis include elevated lesions and necrosis, pseudopodia, corneal ring, endothelial plaque, and hypopyon $[3,5]$. According to the reported literature, the presence of endothelial plaque was considered as a risk factor for lamellar keratoplasty treatment failure [6-8]. However, due to the lack of histopathological evidence, the formation of endothelial plaques is related to anterior chamber reaction of severe fungal infections, or hyphae infiltration of Descemet's membrane remains unclear. Furthermore, it is often difficult to choose deep anterior lamellar keratoplasty (DALK) or penetrating keratoplasty $(\mathrm{PK})$ when encountering endothelial plaques during keratoplasty surgery. In this study, we attempted to use histological evidence to show that endothelial plaques are a reliable sign of hyphae infiltration of Descemet's membrane, thus providing surgical guidance in these circumstances.

\section{Methods}

2.1. Patients. We adhered to the principles outlined in the Declaration of Helsinki, and this study was approved by the ethics committee of Shandong Eye Hospital. A total number of 242 patients with fungal keratitis underwent keratoplasty between January 2013 and March 2017, including DALK for 
89 patients, and PK for 153 patients were reviewed retrospectively. The inclusion criteria were as follows: (1) the hyphae were detected by corneal smear examination or laser scanning confocal microscopy (Heidelberg Instruments, GmbH, Heidelberg, Germany); (2) over 4/5 of the corneal thickness was infected or infiltrated as observed by slit-lamp microscopy, laser scanning confocal microscopy, and anterior segment optical coherence tomography (As-OCT); (3) antifungal medication as reported in our previous studies $[9,10]$ was given for at least 2 weeks but was ineffective. The patients detected with no endothelial plaque and diagnosed with perforation were excluded from this study. Finally, a total of 60 patients (60 eyes) were included ( 26 men and 34 women). Their mean age was 40.5 years (range $31-68$ years).

A comprehensive eye examination was performed with a slit-lamp, including measuring the size of fungal ulcer and the depth of hypopyon. The methods were as follows. Photos of the corneas were obtained with a digital camera at the slitlamp (Topcon, DC-3), and a picture of a graduated scale under the same magnification ratio was taken. Then, the pictures of the corneas and the graduated scale were opened in Adobe Photoshop software. After dragging the graduated scale to the cornea with the move tool, the size of fungal ulcer and the depth of hypopyon were measured and recorded.

2.2. Endothelial Plaque Evaluation. All the surgeries were planned as DALK preoperatively, and the decision of performing DALK or PK was made according to the evaluation of endothelial plaques after exposure of Descemet's membrane with the big-bubble technique. All surgeries were performed by a single surgeon (H.G.). The detailed surgical procedure was introduced in our previous report [11]. After Descemet's membrane was exposed, the endothelial plaques were assessed under the surgical microscope and graded as follows: grade 1, 1-3 endothelial plaques; grade 2, 4-8 endothelial plaques; and grade 3 , more than 8 endothelial plaques or dense, merging endothelial plaques. If only 1-3 endothelial plaques (grade 1) were visible, DALK was performed. If more than 3 endothelial plaques (grade 2-3) were visible, $\mathrm{PK}$ was performed instead. After endothelial plaque evaluation, patients with endothelial plaques of grade 1 continued the surgery as DALK, and those with grades 2 and 3 were converted to $\mathrm{PK}$.

After surgery, the diseased Descemet's membrane and the corneal lamellar tissue were sent for fungal pathogen culture and histopathological examination with calcofluor white and periodic acid-Schiff (PAS) staining.

\subsection{Calcofluor White Staining of Descemet's Membrane.} After PK, Descemet's membranes were stained with calcofluor white staining. Briefly, a drop of $1 \%$ calcofluor white (Sigma, St. Louis, USA) was added to Descemet's membranes obtained during PK, which were incubated for $2 \mathrm{~min}$ at room temperature and washed three times in normal saline. Fungal hyphae were observed using a fluorescence E800 microscope (Nikon, Tokyo, Japan). With calcofluor white staining, the fungal hyphae were bright blue against a dark background.
2.4. Histopathological Examination and Immunohistochemical Detection. Corneal buttons obtained during DALK or PK surgery were fixed in $4 \%$ formalin. These corneal buttons were half-cut along the central line. Serially graded ethanol baths followed by xylene were used to dehydrate the tissues before they were immersed in paraffin wax. The samples were embedded in paraffin molds, and serial slices $(4 \mu \mathrm{m})$ were stained with PAS stain (Maxin, Fujian, China). The hyphae were observed by light microscopy (Olympus BX60, Tokyo, Japan). Six microscopic fields $(\times 400)$ were randomly chosen in each cornea, and the number of fungal hyphae and inflammatory cells per field were counted for the statistical analysis.

The expressions of a neutrophil marker (CD15) and a macrophage marker (CD68) were detected with immunohistochemical staining. Briefly, $4 \mu \mathrm{m}$-sections were obtained from the paraffin-embedded corneal buttons. The antigens were recovered by microwaving the sections, and the endogenous peroxidase activity was quenched with a $3 \%$ solution of hydrogen peroxide for $10 \mathrm{~min}$. The sections were incubated with primary antibodies (mouse anti-CD15 or CD68; Maxin, Fujian, China) for $60 \mathrm{~min}$ at $37^{\circ} \mathrm{C}$ after which they were incubated with HRP-conjugated goat anti-mouse IgG for $30 \mathrm{~min}$ at $37^{\circ} \mathrm{C}$. The peroxidase activity was visualized by incubating the sections in a solution of diaminobenzidine (DAB; Maxin, Fujian, China). Negative controls were performed in the absence of primary antibodies. Finally, the samples were mounted and examined under a microscope (Olympus BX60, Tokyo, Japan).

2.5. Statistical Analysis. All data were analyzed with SPSS software (version 17.0, SPSS Inc., Chicago, IL, USA). Student's $t$ test was used to compare the hyphae and inflammatory cell counts between patients with grade 2 and grade 3 endothelial plaques. The data are shown as the mean\pm standard deviation, and a $P$ value $<0.05$ was considered statistically significant.

\section{Results}

3.1. Patient Information. $\mathrm{KOH}$ smears and laser scanning confocal microscopy were hyphae positive in all 60 patients. The mean size of fungal ulcer was $6.9 \pm 0.6 \mathrm{~mm} \times 6.2 \pm 0.5 \mathrm{~mm}$ before surgery. Hypopyon was present in 38 eyes, measuring $1.5 \pm 0.9 \mathrm{~mm}$ (range: $0.2-4 \mathrm{~mm})$.

3.2. Endothelial Plaque Evaluation and Surgery. All 60 patients had a varying degree of endothelial plaques, including 3 patients in grade 1, 29 patients in grade 2, and 28 patients in grade 3 . The 3 patients with grade 1 underwent DALK surgery, whereas 57 patients with grade 2 and grade 3 underwent PK surgery (Figure 1). After the surgery, the mean follow-up time was $4.1 \pm 2.6$ months (range: 3-6 months). 


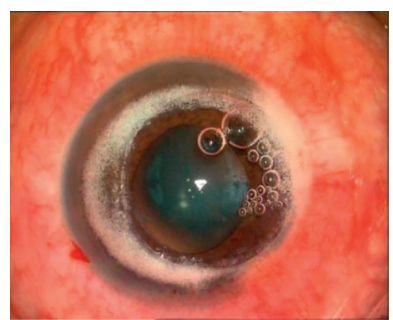

(a)

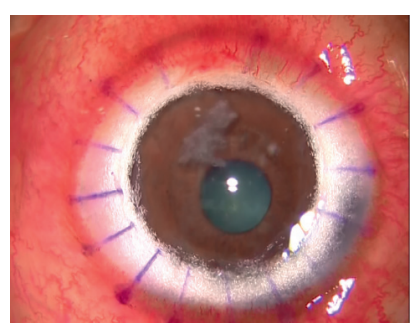

(b)

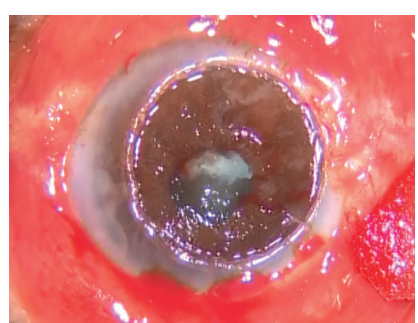

(c)

FIGURE 1: Representative pictures of grade 1 (a), grade 2 (b), and grade 3 (c) endothelial plaques after exposing Descemet's membrane during keratoplasty surgery.

3.3. Fungal Pathogen Distribution. In 38 fungal pathogens positively cultured, 24 (63.2\%) were identified as Aspergillus species, $10(26.3 \%)$ were Fusarium species, 2 (5.2\%) were Alternaria species, and 2 (5.2\%) were Colletotrichum species. There were also 22 unidentified species.

3.4. Calcofluor White Stain Evaluation. With calcofluor white stain, 29 patients with grade 2 and 28 patients with grade 3 were all hyphae positive in Descemet's membranes. The hyphae had many branches and were bamboo-structured and surrounded with inflammatory cells. The representative hyphae in Descemet's membranes of patients with grade 2 and grade 3 are shown in Figure 2.

\subsection{Histopathology and Immunohistochemical Evaluation.} PAS staining the corneal stroma and Descemet's membrane was positive for fungal hyphae in all eyes. In patients with grade 3, the destruction of the cornea stroma was severe, and more hyphae and inflammatory cells were found in the deep stroma and Descemet's membrane. In patients with grade 2 , the inflammation was moderate, and there were less hyphae in the deep stroma and Descemet's membrane (Figure 3). Both the number of hyphae and inflammatory cells per highpower field were significantly different between the patients with grade 3 and grade $2(P<0.01)$ (Figure 4$)$. Immunohistochemical staining of endothelial plaques revealed that most cells were positive for CD15 (neutrophils) or CD68 (macrophages) (Figure 5).

3.6. Fungal Recurrence. Recurrent FK occurred in 2 out of 3 patients who underwent DALK. One eye recurred at 3 days after DALK surgery and at 5 days in another eye. The cultured fungal pathogens were both Aspergillus species in the 2 patients. No fungal recurrence was observed after PK surgery.

\section{Discussion}

The big-bubble-DALK was reported to be effective in the treatment of fungal keratitis. However, during the DALK surgery, if endothelial plaques were present, the doctor often faced a dilemma over whether to continue the DALK procedure or choose the PK procedure. There have been disputes about the possible correlation between hyphae infiltration and endothelial plaques [12-15]. Some researchers believed that the hyphae may invade the deep corneal stroma to cause an anterior chamber reaction as well as endothelial plaques with the progression of the disease, but no direct pathologic evidence was available $[16,17]$. Therefore, it is necessary to study the components of corneal endothelial plaques and their relationship to hyphae penetrating Descemet's membrane, which may help clinical doctors to make an informed choice for surgical procedures.

Corneal endothelial plaques in FK contain a large number of acute inflammatory and immune cells. Our immunohistochemical staining revealed that the corneal endothelial plaques were mainly composed of neutrophils and macrophages surrounding the penetrating hyphae. This structure reflects the body's defense system against invading microorganisms such as the hyphae penetrating into the anterior chamber. Kiryu et al. [18] found that hyphae surrounded by neutrophils showed double or triple cell wall formation and sometimes a hypha-in-hypha structure in dexamethasone-treated corneal lesions. This special structure was regarded as a protective device for the survival of Fusarium species to evade the host's immune system.

Calcofluor white stain is an easy and direct staining method for fungi and is more sensitive for detecting fungal hyphae than the traditional PAS stain on paraffin sections. With direct calcofluor white stain, all patients had hyphae distribution in Descemet's membrane. Histological examination further verified that more hyphae and inflammatory cells were found in the grade 3 patients than in the grade 2 patients with PAS stain. Out of 3 patients with grade 1 endothelial plaques who underwent DALK, 2 patients had recurrent FK after DALK, further suggesting the close relationship between endothelial plaques and hyphae infiltration. These results indicate that endothelial plaques are a reliable sign of hyphae infiltration of Descemet's membrane.

The patients with $4 / 5$ of the corneal thickness infected or infiltrated by hyphae and antifungal medication ineffective for 2 weeks are suggested to undergo keratoplasty. The exact procedure selection (DALK or PK) depends on the evaluation of deeper stroma and Descemet's membrane during the surgery. Surgeons can proceed with DALK when no hyphae and endothelial plaques are detected in the deeper stroma and Descemet's membrane. In a previous study by Dr. Gao [11], DALK was performed in a series of 23 patients with no hyphae in the posterior stroma near Descemet's 


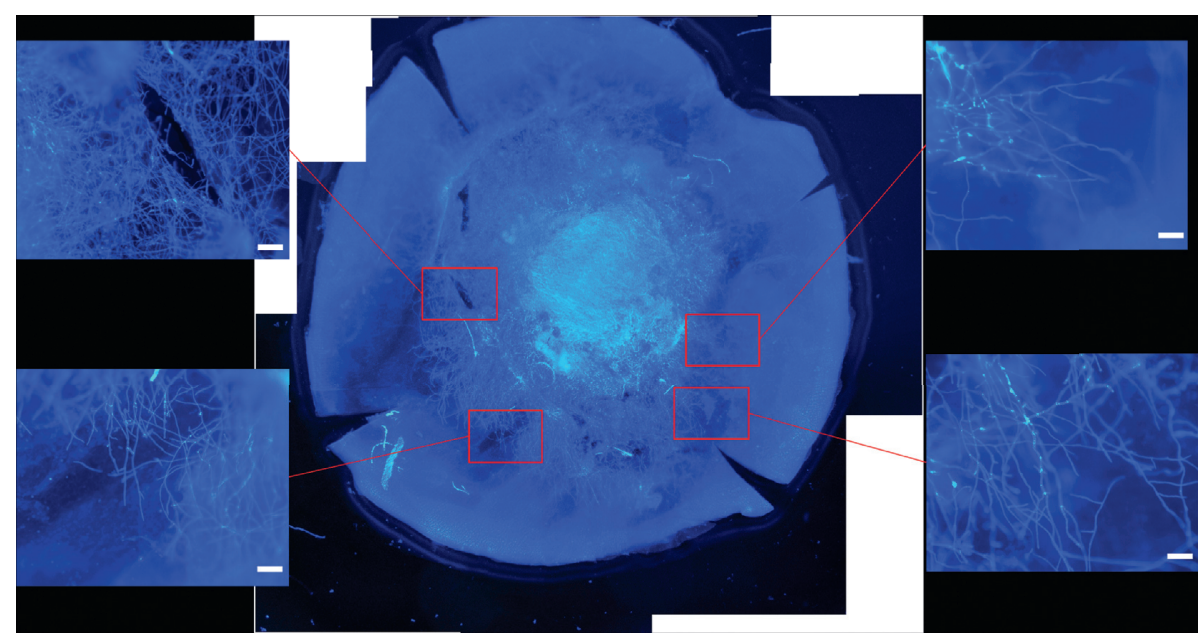

FIGURE 2: In a patient with grade 3 endothelial plaques, fungal hyphae were found in Descemet's membrane with calcofluor white stain by fluorescence microscopy (scale bar $20 \mu \mathrm{m}$ ).

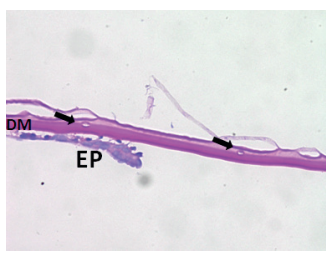

(a)

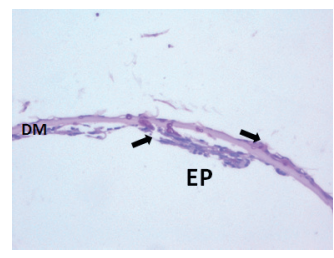

(b)

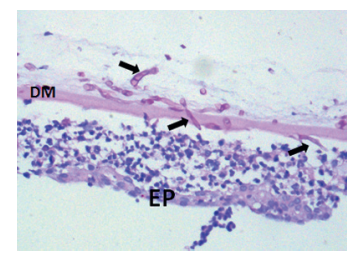

(c)

Figure 3: Hyphae (arrows) were found in patients with grade 1 (a), grade 2 (b), and grade 3 (c) with periodic acid-Schiff (PAS) stain. More hyphae and inflammatory cells were found in Descemet's membrane in patients with grade 3 (c) (scale bar $20 \mu \mathrm{m}$ ).

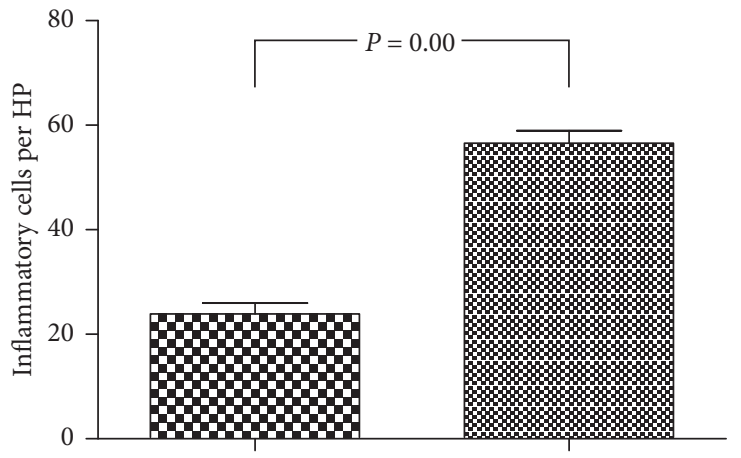

Grade 2
Grade 3

(a)

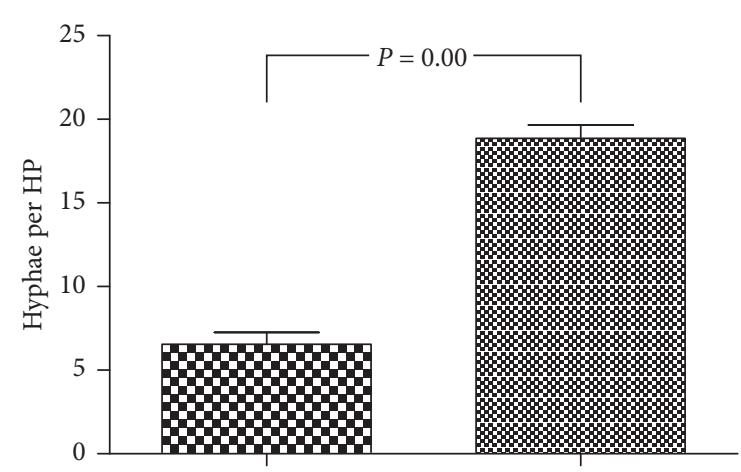

Grade 2

(b)

Figure 4: Significant differences were found between patients with grade 2 and grade 3 endothelial plaques $(P<0.01)$ including $(a)$ the number of inflammatory cells and (b) hyphae present in Descemet's membrane per high-power field.

membrane, and recurrence of FK was found in only two patients $(8.7 \%)$, much lower than the reported values $[13,19,20]$.

Fusarium is still the most common pathogen of fungal keratitis, followed by Alternaria and Aspergillus species, in Shandong Province, China [11, 21-23]. However, in this study, the postoperative fungal pathogen culture of the diseased Descemet's membrane showed that 24 (63.2\%) out of the 38 positively cultured were identified as Aspergillus species. In addition, the cultured pathogens from the 2 patients with recurrent FK after DALK surgery were Aspergillus species. The main reason is that unlike Fusarium species with horizontally growing hyphae, most Aspergillus hyphae grow vertically in corneal stroma, so the deep stroma and Descemet's membrane are prone to be invaded. The rudimentary hyphae in the deep stroma and Descemet's 


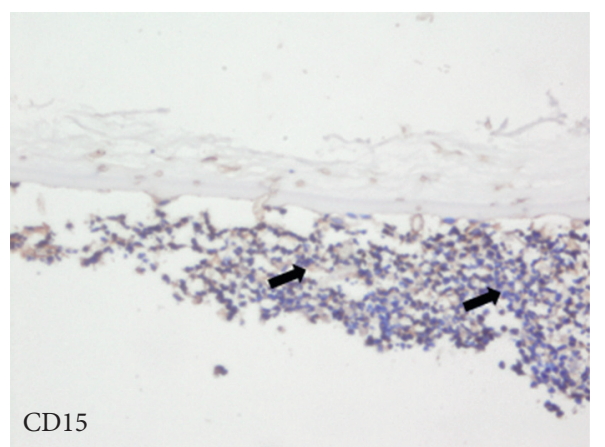

(a)

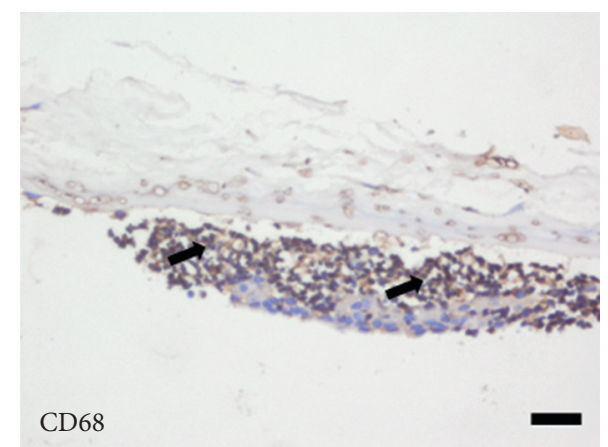

(b)

FIGURE 5: Immunohistochemical staining revealed CD15-positive neutrophils (brown color, arrowed in (a)) and CD68-positive macrophages (brown color, arrowed in (b) accumulated in the endothelial plaques (scale bar $20 \mu \mathrm{m}$ ).

membrane that are not cleared by DALK may cause fungal recurrence postoperatively. Hence, PK should be performed once plaques are detected in endothelium during the surgery, which means hyphae has penetrated Descemet's membrane to reduce the risk of fungal recurrence.

In conclusion, endothelial plaques are considered as a sign of hyphae infiltrating Descemet's membrane. The predominating fungal pathogens of diseased Descemet's membrane were Aspergillus species (63.2\%). Once plaques are detected in endothelium during the surgery, PK should be performed to reduce the risk of fungal recurrence.

\section{Data Availability}

All data and material are available within the article.

\section{Additional Points}

Synopsis. Corneal endothelial plaques in FK contain a large number of bamboo-like hyphae and inflammatory cells. Aspergillus species is the predominating fungal pathogens capable of penetrating Descemet's membrane readily.

\section{Ethical Approval}

This study was approved by the Institutional Review Board of Shandong Eye Hospital, Jinan, China.

\section{Conflicts of Interest}

The authors declare that they have no conflicts of interest.

\section{Authors' Contributions}

Xiaolin Qi and Ting Liu contributed equally to this work.

\section{Acknowledgments}

The authors sincerely thank their colleagues at Shandong Eye Hospital for their valuable discussion and help. This study was supported by the National Natural Science Foundation of China (81870639 and 81900907), Key Project of National Natural Science Foundation of China
(81530027), Taishan Scholar Program (20150215, 201812150), and Innovation Project of Shandong Academy of Medical Sciences (2019RC009).

\section{References}

[1] J. Sengupta, A. Khetan, S. Saha, D. Banerjee, N. Gangopadhyay, and D. Pal, "Candida keratitis," Cornea, vol. 31, no. 4, pp. 371-375, 2012.

[2] A. K. Ghosh, A. Gupta, S. M. Rudramurthy, S. Paul, V. K. Hallur, and A. Chakrabarti, "Fungal keratitis in North India: spectrum of agents, risk factors, and treatment," Mycopathologia, vol. 181, no. 11-12, pp. 843-850, 2016.

[3] P. Garg, A. Roy, and S. Roy, "Update on fungal keratitis," Current Opinion in Ophthalmology, vol. 27, no. 4, pp. 333339, 2016.

[4] L. Xie, W. Zhong, W. Shi, and S. Sun, "Spectrum of fungal keratitis in north China," Ophthalmology, vol. 113, no. 11, pp. 1943-1948, 2006.

[5] P. A. Thomas, A. K. Leck, and M. Myatt, "Characteristic clinical features as an aid to the diagnosis of suppurative keratitis caused by filamentous fungi," British Journal of Ophthalmology, vol. 89, no. 12, pp. 1554-1558, 2005.

[6] L. Xie, J. Hu, and W. Shi, "Treatment failure after lamellar keratoplasty for fungal keratitis," Ophthalmology, vol. 115, no. 1, pp. 33-36, 2008.

[7] D. G. Said, M. Otri, A. Miri, A. Kailasanathan, T. Khatib, and H. S. Dua, "The challenge of fungal keratitis," British Journal of Ophthalmology, vol. 95, no. 12, pp. 1623-1624, 2011.

[8] Y. Takezawa, T. Suzuki, and A. Shiraishi, "Observation of retrocorneal plaques in patients with infectious keratitis using anterior segment optical coherence tomography," Cornea, vol. 36, no. 36, pp. 1237-1242, 2017.

[9] L. Xie, X. Dong, and W. Shi, "Treatment of fungal keratitis by penetrating keratoplasty," British Journal of Ophthalmology, vol. 85, no. 9, pp. 1070-1074, 2001.

[10] L. Xie, W. Shi, Z. Liu, and S. Li, "Lamellar keratoplasty for the treatment of fungal keratitis," Cornea, vol. 21, no. 1, pp. 33-37, 2002.

[11] H. Gao, P. Song, J. J. Echegaray et al., "Big-bubble deep anterior lamellar keratoplasty for management of deep fungal keratitis," Journal of Ophthalmology, vol. 2014, Article ID 209759, 8 pages, 2014.

[12] S.-P. Chee and A. Jap, "Immune ring formation associated with cytomegalovirus endotheliitis," American Journal of Ophthalmology, vol. 152, no. 3, pp. 449-453, 2011. 
[13] A. Anshu, A. Parthasarathy, J. S. Mehta, H. M. Htoon, and D. T. H. Tan, "Outcomes of therapeutic deep lamellar keratoplasty and penetrating keratoplasty for advanced infectious keratitis," Ophthalmology, vol. 116, no. 4, pp. 615-623, 2009.

[14] X. Dong, W. Shi, Q. Zeng, and L. Xie, "Retracted: roles of adherence and matrix metalloproteinases in growth patterns of fungal pathogens in cornea," Current Eye Research, vol. 30, no. 8, pp. 613-620, 2005.

[15] G. Galperin, M. Berra, J. Tau, G. Boscaro, J. Zarate, and A. Berra, "Treatment of fungal keratitis from Fusarium infection by corneal cross-linking," Cornea, vol. 31, no. 2, pp. 176-180, 2012.

[16] A. Konstantopoulos, J. Kuo, D. Anderson, and P. Hossain, "Assessment of the use of anterior segment optical coherence tomography in microbial keratitis," American Journal of Ophthalmology, vol. 146, no. 4, pp. 534-542, 2008.

[17] R. Shetty, H. Nagaraja, C. Jayadev, Y. Shivanna, and T. Kugar, "Collagen cross-linking in the management of advanced nonresolving microbial keratitis," British Journal of Ophthalmology, vol. 98, no. 8, pp. 1033-1035, 2014.

[18] H. Kiryu, S. Yoshida, Y. Suenaga, and M. Asahi, "Invasion and survival of Fusarium solani in the dexamethasone-treated cornea of rabbits," Journal of Medical and Veterinary Mycology, vol. 29, no. 6, pp. 395-406, 1991.

[19] S.-E. Ti, J. A. Scott, P. Janardhanan, and D. T. H. Tan, "Therapeutic keratoplasty for advanced suppurative keratitis," American Journal of Ophthalmology, vol. 143, no. 5, pp. 755-762, 2007.

[20] W. Shi, T. Wang, L. Xie et al., "Risk factors, clinical features, and outcomes of recurrent fungal keratitis after corneal transplantation," Ophthalmology, vol. 117, no. 5, pp. 890-896, 2010.

[21] L. Xie, H. Zhai, W. Shi, J. Zhao, S. Sun, and X. Zang, "Hyphal growth patterns and recurrence of fungal keratitis after lamellar keratoplasty," Ophthalmology, vol. 115, no. 6, pp. 983-987, 2008.

[22] L. Xie, H. Zhai, J. Zhao, S. Sun, W. Shi, and X. Dong, “Antifungal susceptibility for common pathogens of fungal keratitis in Shandong Province, China," American Journal of Ophthalmology, vol. 146, no. 2, pp. 260-265, 2008.

[23] Q. Wang, D. Luo, H. Chen, and X. Sun, "The distribution and drug resistance rate of 3759 cases of fungal keratitis," Chinese Journal of Mycology, vol. 14, pp. 16-19, 2019. 